\title{
DIAGNÓSTICO AMBIENTAL DAS MARISMAS NO ESTUÁRIO DA LAGOA DOS PATOS - RS
}

\author{
JULIANO CÉSAR MARANGONI ${ }^{1,2} \&$ CÉSAR SERRA BONIFÁCIO COSTA ${ }^{1}$ \\ 1 - Universidade Federal do Rio Grande - Instituto de Oceanografia. Pós-Graduação em Oceanografia Biológica. Laboratório de Ecologia \\ Vegetal Costeira. Instituto de Oceanografia. Caixa.Postal, 474, Rio Grande - .RS - CEP 96201-900 - Brasil. 2 - julianomarangoni@pop.com.br
}

\begin{abstract}
As marismas no estuário da Lagoa dos Patos vem sendo suprimidas desde o século XIX pela expansão urbana, portuária e industrial, entretanto, nos últimos 53 anos perdas de área foram causadas principalmente por processo natural (erosão). As atividades antrópicas no entorno das marismas vem causando modificações na cobertura vegetal, afetando processos biológicos e ecológicos. Este estudo visou identificar e quantificar os efeitos adversos das perturbações de origem antrópica (pastejo, fogo, corte da vegetação, lixo, construção de aterros e canais de drenagem) e de origem natural (erosão) sobre as marismas. As perturbações antrópicas e erosão foram quantificadas em intensidade e extensão, avaliadas através de transeções aleatórias. As marismas no estuário da Lagoa dos Patos apresentam algum tipo de perturbação que afetam a vegetação com baixa intensidade e moderada extensão $(10-50 \mathrm{~m})$. Das 23 unidades de marismas avaliadas foi possível detectar 4 grupos de unidades sob dominância de perturbações causadas pela agropecuária (11 unidades-pastejo), atividade urbana (5 unidades-lixo), fogo (4 unidades) e erosão (3 unidades). As marismas demonstram uma boa taxa de recuperação da cobertura vegetal ( 3 anos) após as perturbações, fazendo com que este ecossistema seja resiliente frente aos impactos ambientais avaliados (pastejo, fogo, lixo e corte da vegetação).
\end{abstract}

RESUMO

PALAVRAS-CHAVE: Marismas, diagnóstico ambiental, atividades antrópicas, erosão

\section{ABSTRACT}

Environmental Assessment of saltmarshes at Patos Lagoon Estuary - RS

Saltmarshes in Patos Lagoon Estuary have been converted since XIX century due to urban, port and industrial expansion, however in the last 53 years losses of saltmarshes were caused mainly by natural process (erosion). Biological and ecological processes in saltmarshes have been modified by anthropogenic activities, so this study aimed to identify and quantify the anthropogenic (grazing, fire, harvesting, litter, embankments, and man-made channels) and natural disturbances (erosion) on saltmarshes. Anthropogenic disturbances and erosion were quantified in intensity and spatial extension, evaluated through random transects. Saltmarshes showed some types of disturbance (anthropogenic and/or erosion) with low intensity and moderate extension $(10-50 \mathrm{~m})$. There are 23 saltmarsh units at Patos Lagoon Estuary, and it was possible to detect 4 main groups of units under different disturbances, caused by farming and ranching (11 unit-grazing), urban activities (5 unit-litter), fire ( 4 units), and erosion ( 3 units). Saltmarsh plants have showed good resilience by fast recovering ( 3 years) after anthropogenic disturbances (grazing, fire, litter and harvesting).

KEY-WORDS: Saltmarshes, environmental assessment, anthropogenic activities, erosion

\section{INTRODUÇÃO}

As marismas ocupam $70 \mathrm{~km}^{2}$ de áreas entremarés nas margens e ilhas no estuário da Lagoa dos Patos (RS) (Costa \& Davy 1992, Costa et al. 1997, Nogueira \& Costa 2003, Marangoni 2008). Este ecossistema oferece abrigo e hábitats para várias espécies de animais estuarinos e costeiros, sendo sua produção primária um importante componente da teia trófica na região estuarina (Costa et al. 1997, Seeliger et al. 1998, Costa 1998, Bemvenuti 1998, Seeliger 2001, Abreu et al. 2006).

Vários trabalhos vêm relatando a degradação destas marismas por atividades antrópicas como o pastejo por animais domésticos, incêndios, corte da vegetação, deposição de lixo, construção de aterros e canais de drenagem (Costa 1997, Costa et al. 1997, Seeliger \& Costa 1998, Costa \& Marangoni 2000, Marangoni 2003). Estes impactos sobre a cobertura vegetal podem afetar os processos biológicos e ecológicos nas marismas, tais como a produção de biomassa (Hackney \& De La Cruz 1981, Bazely \&
Jefferies 1985), reprodução das plantas (Turner 1987, Dormann \& Bakker 2000), diversidade de espécies (Brewer et al. 1998, Bos et al. 2002, Bakker et al. 2003, Feldman \& Lewis 2005, Madanes et al. 2007), disponibilidade de nutrientes (Bazely \& Jefferies 1985), composição e abundância de fauna (Isacch et al. 2004).

Costa \& Marangoni (2000) definem como impactos estruturais os efeitos adversos das perturbações antrópicas e natural (erosão) que afetam a integridade da cobertura vegetal das marismas. Alterações estruturais do desenvolvimento e composição da cobertura vegetal podem ser estimadas visualmente, considerando (1) a escolha das perturbações (antrópicas e natural) que podem ser identificadas e diferenciadas das demais causas, bem como avaliadas visualmente e (2) 0 desenvolvimento de uma escala objetiva para quantificação da severidade da perturbação. Esta metodologia constitui uma técnica de avaliação de impacto ambiental, cujos principais aspectos são a facilidade e a rapidez no processo de quantificação 
das alterações ambientais. Esta técnica foi empregada pontualmente em avaliações de impacto ambiental (Costa \& Marangoni 2000) sobre as marismas, entretanto, nenhum estudo englobando todo o estuário da Lagoa dos Patos foi ainda publicado.

Na região estuarina da Lagoa dos Patos existem 24 áreas de marisma espacialmente distintas conforme a descontinuidade física ou cobertura vegetal (Costa et al. 1997, Nogueira \& Costa 2003), compreendendo os municípios costeiros de Rio Grande e São José do Norte, doravante designados, respectivamente, RG e SJN. Este estudo teve como objetivo identificar e quantificar as perturbações antrópicas e natural (erosão) sobre as marismas no estuário da Lagoa dos Patos a partir de alterações da cobertura vegetal das marismas, visando subsidiar 0 processo de gerenciamento ambiental deste ecossistema.

\section{MATERIAL E MÉTODOS}

\section{Área de Estudo}

As marismas se restringem à região inferior do estuário da Lagoa dos Patos (Costa et al. 1997), tendo como limite continental de distribuição o canal de São Gonçalo na margem oeste e Ponta Rasa na margem leste (3150' - 32응' Este estudo foi efetuado em 23 das 24 unidades de marismas do estuário da Lagoa dos Patos nos municípios de RG (margem oeste) e SJN (margem leste). A ilha do Terrapleno (unidade 10 sensu Nogueira \& Costa 2003) inclui marismas desenvolvidas sobre material dragado depositado na década de 1970 pelo Porto de Rio Grande e, suas condições ambientais não foram avaliadas porque serão removidas para ampliação da zona de manobra das embarcações (Figura 1).

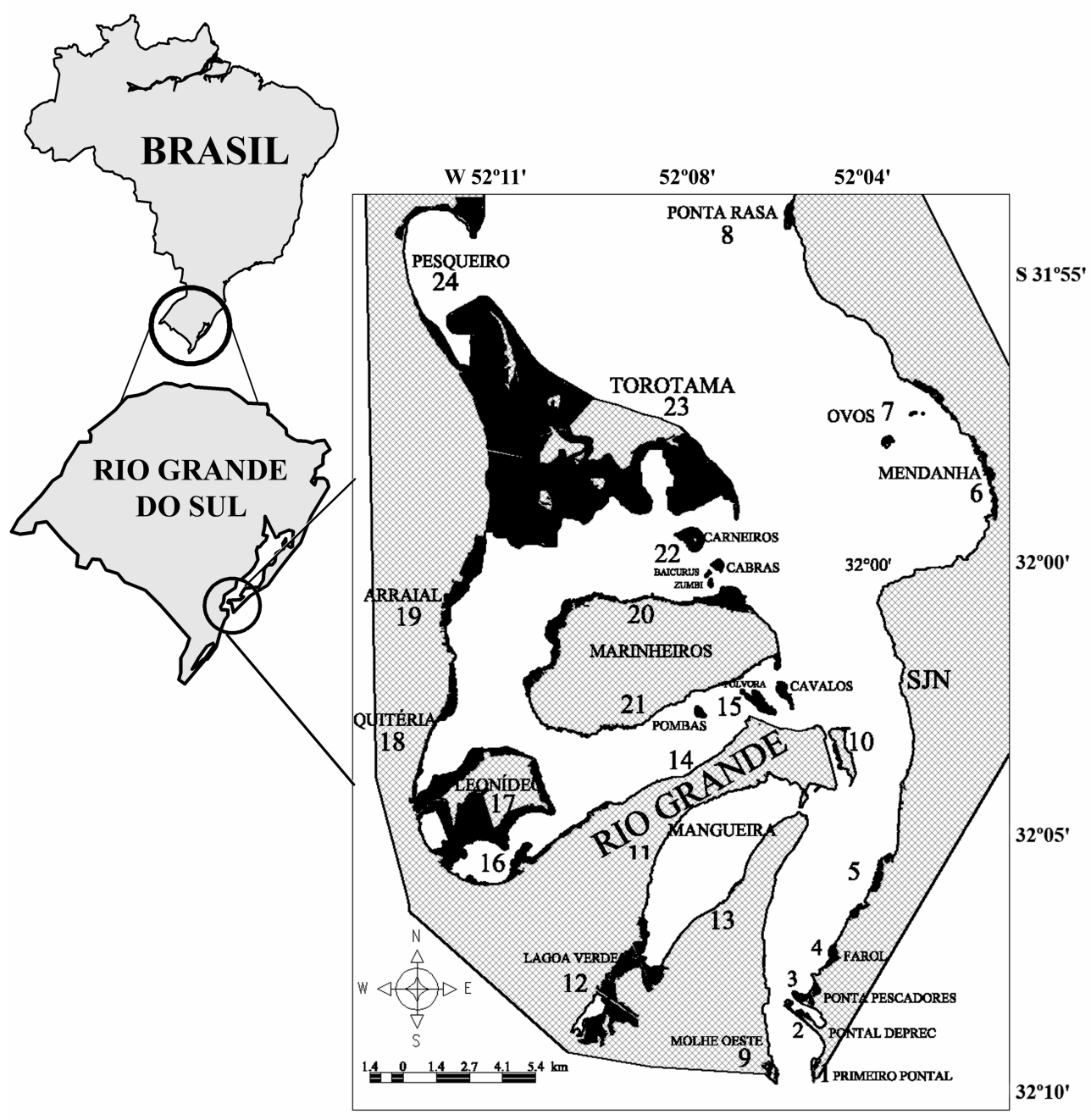

Figura 1. Estuário da Lagoa dos Patos (RS - Brasil) com a localização das 24 unidades de marismas (áreas Escuras) nos municípios de Rio Grande e São José do Norte. 


\section{Avaliação Ambiental}

No ano de 2005 (de janeiro a novembro) realizou-se a estimativa visual das perturbações antrópicas e natural (erosão) responsáveis por modificações na cobertura vegetal (estrutural) em cada uma das 23 unidades de marismas. A avaliação visual foi efetuada nas marismas inferiores vegetadas principalmente por Spartina alterniflora e Scirpus olneyi (alagadas até $60 \%$ do tempo) (Costa 1998), que ocupam aproximadamente $1 \mathrm{~km}^{2}$ da área total de marismas (Nogueira \& Costa 2003), sobre as marismas média/superiores dominada por Scirpus maritimus, Spartina densiflora e Juncus kraussii (alagadas até $25 \%$ do tempo) (Costa 1998), que colonizam $67 \mathrm{~km}^{2}$ da área total de marismas (Nogueira \& Costa 2003).

Quatro tipos de perturbações de origem antrópica, pastejo por animais domésticos, fogo, corte da vegetação e acúmulo de lixo e uma perturbação natural (erosão das margens) sobre a cobertura vegetal foram quantificados quanto à intensidade e a extensão. Adicionalmente, foi avaliada qualitativamente (presença do impacto) a descontinuidade da cobertura vegetal halófita dominante das marismas por espécies indicadoras de banhado de água doce (mudança da comunidade vegetal), bem como a existência de aterros (deposição de sedimento, rochas, entulhos de construção) e a construção de canais de drenagem.

Para identificar e avaliar os efeitos adversos de origem antrópica e natural sobre as marismas, foram efetuadas transeções aleatórias (localizadas com uso de GPS-UTM Datum SAD69) ao longo de cada uma das 23 unidades da marisma. O número de transeções foi dependente da área e da extensão horizontal deste ecossistema em cada unidade amostrada, mantendo como limite mínimo de amostragem três transeções. Foi efetuado um total de 151 transeções e cada transeção cobriu transversalmente toda a marisma avaliada, do entremarés inferior não vegetado até a transição continental entre a marisma e outro ambiente (e.g. duna, campo, área agrícola ou área urbana). Estas transeções tiveram comprimento que variaram de $<10 \mathrm{~m}$ até $4 \mathrm{~km}$. Concomitantemente as avaliações visuais das perturbações antrópicas e natural, foram registradas as espécies vegetais dominantes da cobertura vegetal e/ou muito freqüente em cada zona entremarés.

Após o percurso de cada transeção, em cada zona entremarés (inferior e média/superior), foram conferidos valores na escala de 0 a 3 , para intensidade e para extensão das perturbações antrópicas de pastejo, fogo, corte da vegetação e acúmulo de lixo, além da erosão, todos estimados a partir da escala abaixo:

Intensidade

$0=$ inexistente;

1 = intensidade baixa;

2 = intensidade moderada;

$3=$ intensidade alta.

$$
\begin{aligned}
& \text { Extensão } \\
& 0=\text { inexistente; } \\
& \begin{array}{l}
1=\text { pequena extensão }(<10 \mathrm{~m}) ; \\
2=\text { extensão moderada }(10-50 \mathrm{~m}) ; \\
3=\text { grande extensão }(>50 \mathrm{~m}) .
\end{array}
\end{aligned}
$$

Nas marismas de pequena largura $(<10 \mathrm{~m})$ foi atribuído valor máximo em extensão (3) quando constatada a existência do impacto ambiental e/ou erosão. Para definir a intensidade da perturbação

\begin{tabular}{|c|c|c|c|c|}
\hline & $\begin{array}{c}\text { Inexistente } \\
0 \\
\end{array}$ & $\begin{array}{c}\text { Intensidade } \\
\text { Baixa } \\
1\end{array}$ & $\begin{array}{c}\text { Intensidade } \\
\text { Moderada } \\
2 \\
\end{array}$ & $\begin{array}{c}\text { Intensidade } \\
\text { Alta } \\
3 \\
\end{array}$ \\
\hline Pastejo & sem marcas & marcas & parcial & total \\
\hline Corte & sem & ------- & ------- & total \\
\hline Fogo & sem vestígios & vestígios & parcial & total \\
\hline Lixo & sem & presença & pouco lixo & muito lixo \\
\hline Erosão & sem & $\begin{array}{l}\text { erosão na marisma } \\
\text { inferior }\end{array}$ & $\begin{array}{l}\text { erosão na marisma } \\
\text { média/superior }\end{array}$ & $\begin{array}{l}\text { escarpa na marisma } \\
\text { média/superior }\end{array}$ \\
\hline
\end{tabular}
antrópica e erosão, foram atribuídos critérios visuais quantitativos das distintas perturbações (Tabela 1).

Tabela 1. Critérios qualitativos para quantificação visual da intensidade das perturbações sobre a cobertura vegetal das marismas do estuário da Lagoa dos Patos. 
Quando da ocorrência de relatos em estudos pretéritos (Costa, 1997; Costa et al. 1997; Costa \& Marangoni 2000; Azevedo 2000; Marangoni 2003) de perturbações antrópicas, ou quando características locais, tais como proximidade de estradas, atividade agropecuária e acampamentos de pescadores, evidenciavam a potencialidade de impacto foram atribuídos valores máximos (3) para intensidade e extensão para a transeção, mesmo não sendo visualizados os impactos ambientais no momento da amostragem.

Para cada unidade, após a quantificação dos impactos ambientais e da erosão (intensidade e extensão), foi calculado o nível médio da perturbação para cada zona entremarés (inferior e média/superior), através da média aritmética dos valores atribuídos a cada item avaliado (pastejo, fogo, corte, lixo e erosão) nas transeções efetuadas (Tabela 2), segundo a equação abaixo:

$$
\underset{\text { Médio }}{\text { Nível }}=\frac{\sum \mathrm{VPi}}{\mathrm{N}}
$$

onde, "VPi" representa os valores das perturbações (pastejo, fogo, corte, lixo e erosão) em Intensidade e em Extensão para cada zona entremarés nas $\mathrm{N}$ transeções.

Tabela 2. Exemplo de uma matriz de avaliação das perturbações antrópicas e natural.

\begin{tabular}{l|cc|cc|}
\hline \multirow{2}{*}{ Transeção 1 } & \multicolumn{2}{|c|}{ Marisma Inferior } & \multicolumn{2}{c|}{$\begin{array}{c}\text { Marisma Média/Superior } \\
\end{array}$} \\
& Intensidade & Extensão & Intensidade & Extensão \\
\hline pastejo & 0 & 0 & 3 & 3 \\
fogo & 0 & 0 & 3 & 3 \\
corte & 0 & 0 & 0 & 0 \\
erosão & 0 & 0 & 0 & 0 \\
acúmulo de lixo & 1 & 3 & 3 & 3 \\
\hline
\end{tabular}

Para cada zona entremarés (inferior e média/superior), em cada unidade da marisma, as perturbações foram hierarquizadas através da média aritmética de todas as transeções dos valores atribuídos em intensidade e extensão. Adicionalmente, o nível médio das perturbações para cada zona entremarés, em cada unidade, foi classificado em uma escala intervalar, sendo considerado nível "inexistente" $=0$, "baixo" $=0,1-1,0$, "moderado"=1,1-2,0 e "severo"= 2,1-3,0.

\section{Análises Estatísticas}

Com a finalidade de verificar a existência de grupos de unidades sob níveis similares de perturbação, foi utilizada uma matriz numérica contendo $\circ$ valor médio das 5 perturbações quantificadas das 23 unidades (agregando as zonas entremarés inferior e média/superior) para Análise de Agrupamento, usando o índice de similaridade Correlação de Pearson e o método de agrupamento UPGMA (Unweighted Pair-Group Method Using Arithmetic Averages) (Romesburg 1984).
Adicionalmente, de forma a caracterizar quais perturbações foram determinantes na formação dos grupos predefinidos (Análise de Agrupamento), foi efetuada a Análise de Correspondência ( $A C)$ com a mesma matriz numérica utilizada na Análise de Agrupamento. A AC é um método de ordenação espacial visando à organização de um conjunto de dados, cuja única exigência é uma matriz numérica com valores positivos (Hair et al. 2005).

Para verificar a significância estatística entre os grupos de unidades sob dominância de diferentes perturbações (oriundos da Análise de Agrupamento), efetuou-se uma análise ANOVA Unifatorial (modelos nulos com 5000 randomizações), utilizando os grupos de unidades como fator e os escores dos 2 eixos principais da AC (coordenadas dos pontos) como as variáveis analisadas. Para a ANOVA de modelo nulo não se faz necessária à normalidade e homocedasticidade dos dados.

As análises estatísticas (Análise de Agrupamento e AC) foram efetuadas pelos programas 
de computador PAST (Hammer 2001) e ANOVA no programa Resampling Procedures 1.3 (Howell 2001).

\section{RESULTADOS}

Todas as marismas no estuário da Lagoa dos

Patos apresentaram algum tipo de perturbação antrópica e/ou natural. A maioria das marismas inferiores teve baixo nível de perturbação (90\%), contrastando com as médias/superiores onde em $65 \%$ das unidades ocorreu um nível moderado de perturbação (Tabela 3).

Tabela 3. Descrição das perturbações sobre as marismas do estuário da Lagoa dos Patos nos municípios de RG e SJN, organizados em Nível da perturbação (INE-Inexistente, BX-Baixo, MOD-Moderado e SEV-Severo), Causas (PAST-Pastejo, FOG-Fogo, CORT-Corte, LIX-Lixo e ERO-Erosão) e Outras perturbações (AT-Aterro/Canais de Drenagem e DES-Descontinuidade da Cobertura Vegetal das Marismas). As causas de perturbações antrópicas e natural estão classificadas em ordem decrescente de importância.

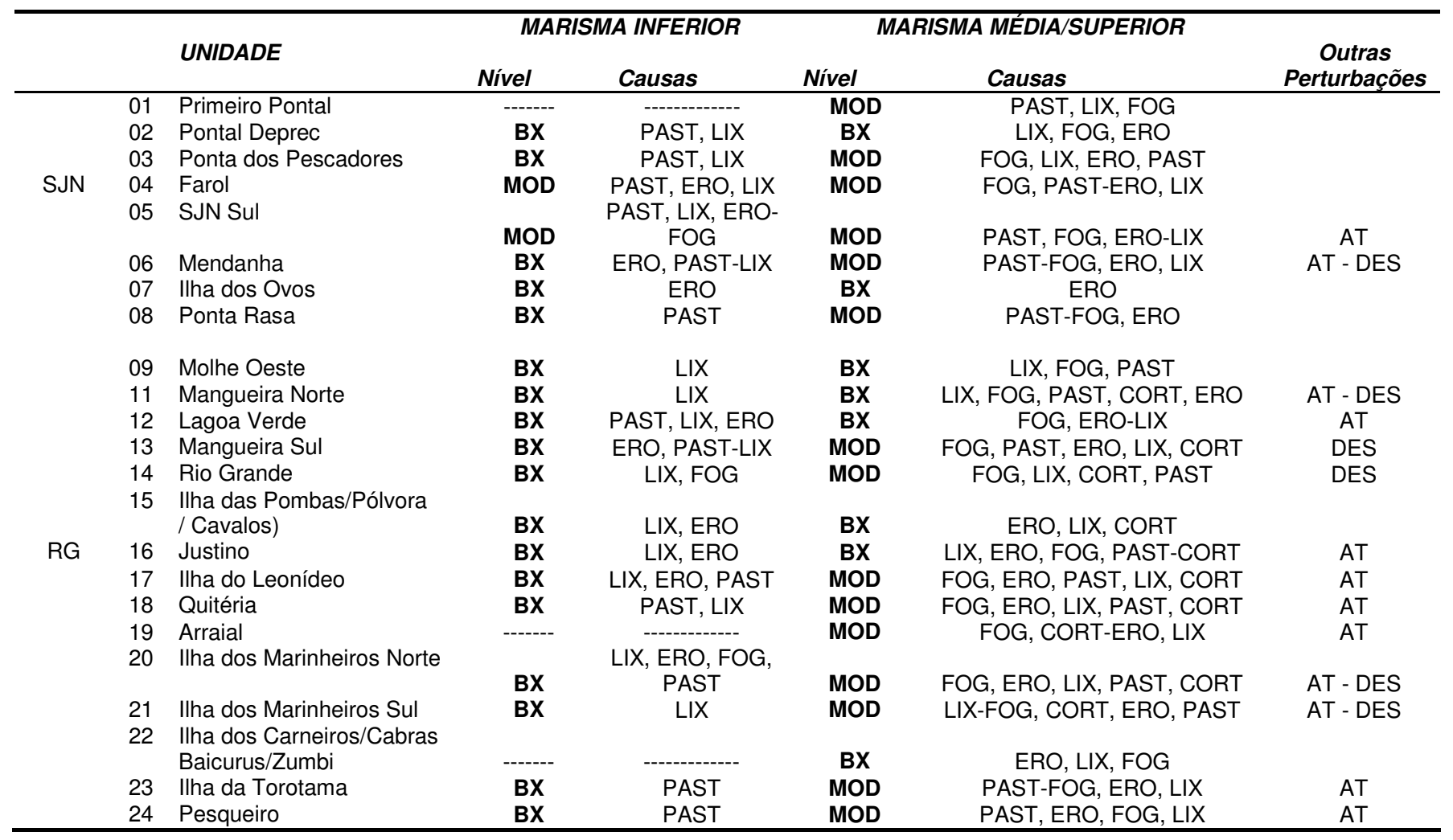

O dendrograma da Análise de Agrupamento demonstrou uma forte descontinuidade, caracterizando 4 grupos de unidades de marismas com uma similaridade intragrupo maior do que $60 \% \mathrm{e}$ dominados por distintos tipos de perturbações. O grupo sob pastejo predominou na maioria das unidades ( $n=11 ; 48 \%)$, com maior influência em SJN (unidades 01, 02, 03, 04, 05, 06 e 08) e secundariamente em RG (unidades 12, 13, 23 e 24). $O$ fogo atuou exclusivamente em RG, mais fortemente nas unidades $17,18,19$ e 20. O acúmulo de lixo afetou predominantemente as unidades 09 , $11,14,16$ e 21 . O grupo sob erosão foi composto pelas unidades 07,15 , e 22. A perturbação por corte da vegetação não foi predominante em nenhuma unidade das marismas (Figura 2). 


\section{Similaridade}

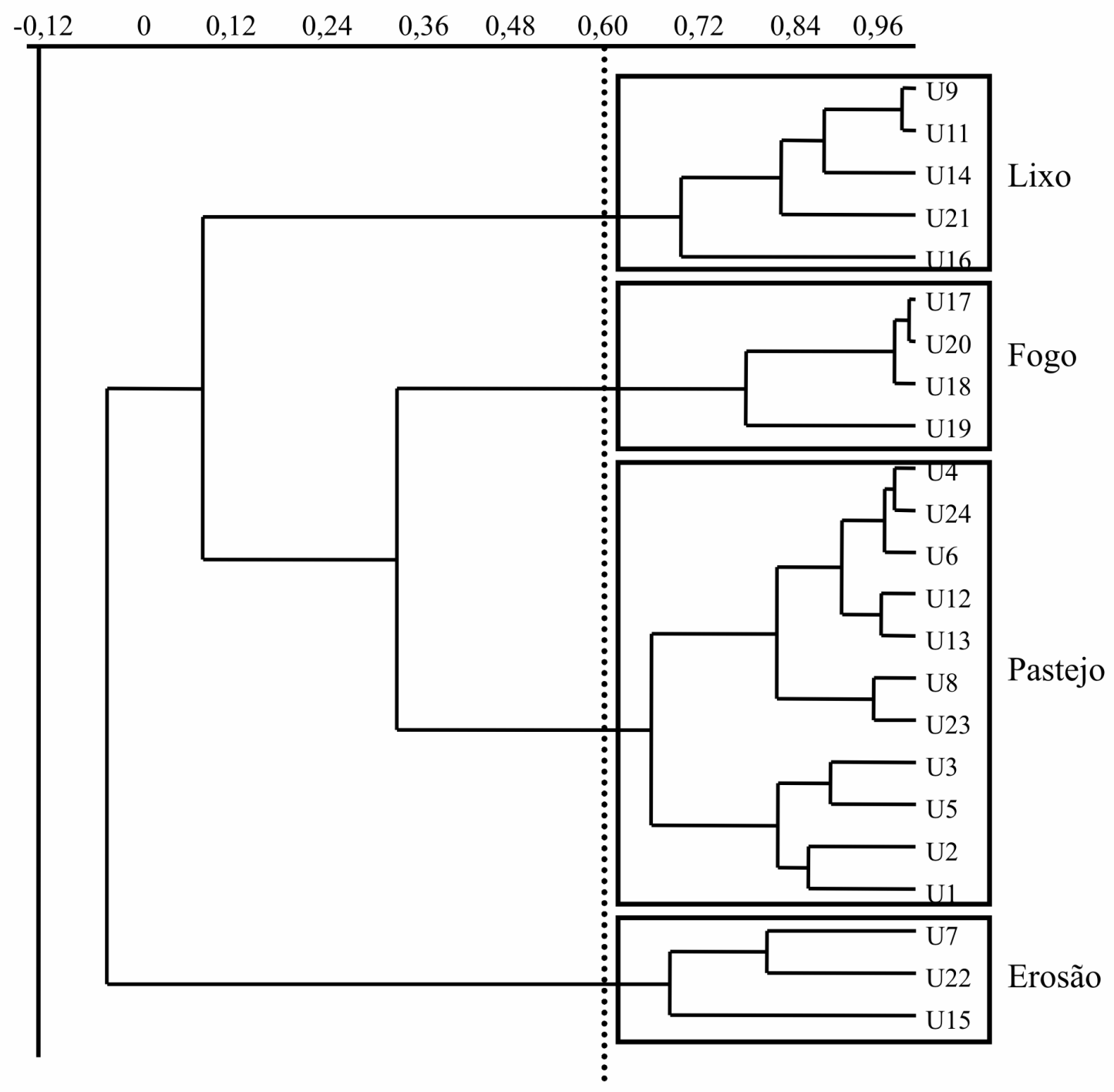

Figura 2. Caracterização dos grupos de unidades de marismas sob similar perturbação, através da Análise de Agrupamento. A linha pontilhada mostra o ponto de corte em $60 \%$ de similaridade. A identificação dos grupos de unidades foi determinada pela dominância da perturbação (maior valor).

A ordenação espacial das unidades de marisma em relação aos níveis médios de perturbação, efetuada pela AC, detectou dois eixos principais que retiveram $41 \%$ (Eixo 1) e $36 \%$ (Eixo 2) da variação da matriz analisada. Os 4 grupos de unidades sujeitos à dominância das distintas perturbações foram discriminados de forma significativa pelo Eixo $1 \quad(p<0,0001)$, uma vez que a ANOVA detectou diferenças entre os seus escores. $O$ Eixo 2 também diferenciou significativamente os 4 grupos de unidades $(p<0,0001)$ (Figura 3$)$. O Eixo 1 $A C$ ordenou as unidades de marismas ao longo de um gradiente de uso da biomassa vegetal, entre áreas preferenciais de pastejo até locais sem pastejo mas com corte da cobertura vegetal por agricultores passando por unidades mais próximas de áreas urbanas com múltiplas perturbações (fogo, lixo, erosão). O Eixo 2 da AC distribuiu as unidades de marisma em função da perturbação sobre as marismas inferiores, que demonstraram ser afetadas pelo acúmulo de lixo em um extremo do eixo e afetadas ou suprimidas pela erosão no outro extremo do eixo. 


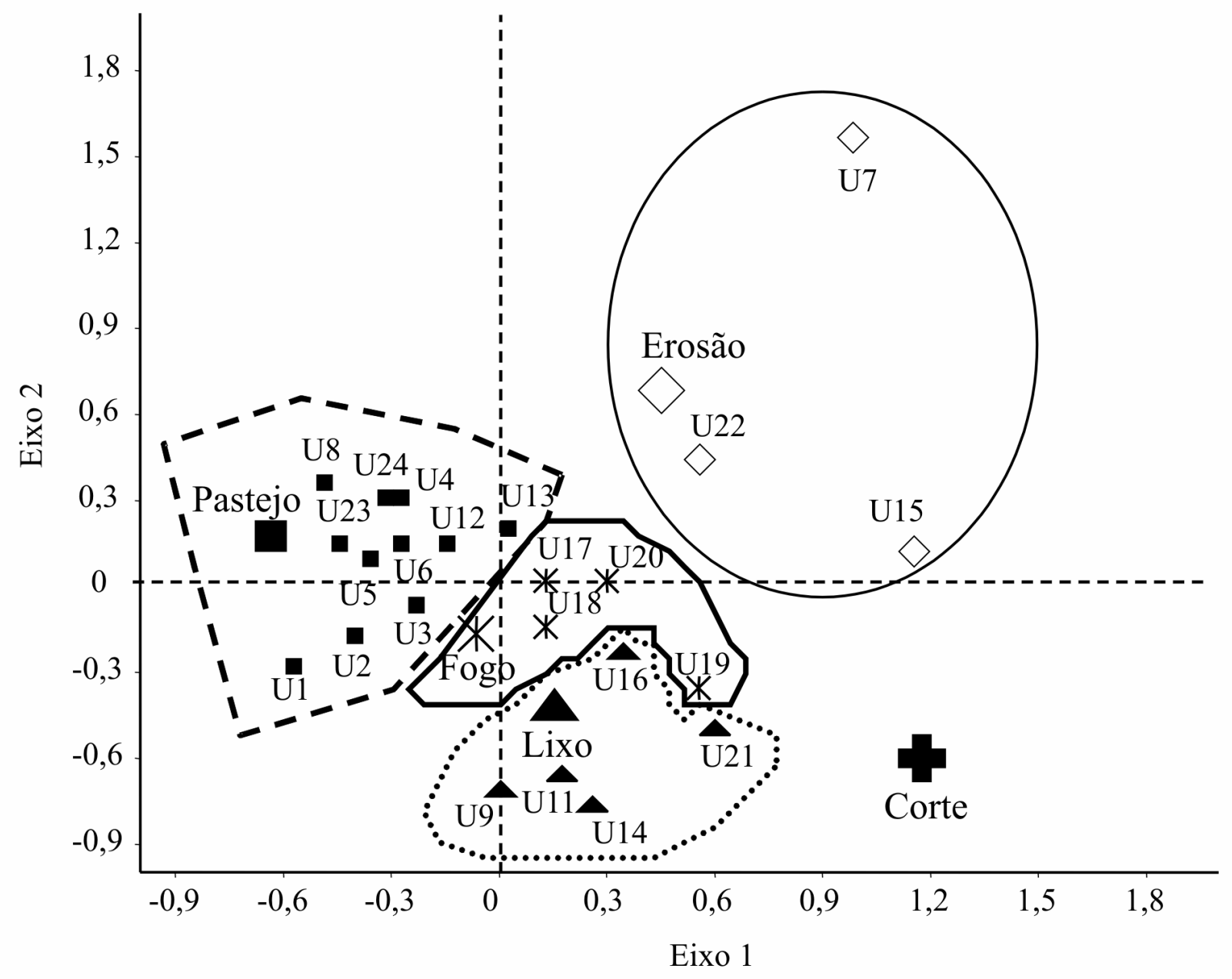

Figura 3. Ordenação espacial dos tipos de perturbação e as unidades de marismas no estuário da Lagoa dos Patos ( $n=23)$ através da Análise de Correspondência.

O pastejo principalmente por bovinos apresentou nível severo de perturbação sobre as unidades 01, 04, 05, 06, 08, 23 e 24 afastadas dos maiores centros urbanos. Estas áreas são vegetadas geralmente por $S$. alterniflora e $S$. olneyi nas marismas inferiores e $S$. densiflora nas zonas médias/superiores (Tabelas 3 e 4) (Figuras 2, 3 e 4). As unidades 01,02 e 03 estão localizadas no entorno urbano (Povoação da Barra - SJN), contudo, como estas marismas possuem livre acesso, os moradores urbanos usam estas áreas como pastagem para gado de leite e cavalos. Em várias unidades sob pastejo foi constatada a ocorrência das gramíneas Paspalum vaginatum e Axonopus sp. (unidades 01, 02, 03, 06, 08 e 24) (Tabela 4). 
Tabela 4. Lista das plantas com maior freqüência de ocorrência observadas nas zonas entremarés inferior e média/superior das marismas no estuário da Lagoa dos Patos (RS, Brasil).

\begin{tabular}{|c|c|c|c|c|}
\hline & & UNIDADE & $\begin{array}{l}\text { MARISMA } \\
\text { INFERIOR }\end{array}$ & $\begin{array}{c}\text { MARISMA } \\
\text { MÉDIA/SUPERIOR }\end{array}$ \\
\hline \multirow{8}{*}{ SJN } & 01 & Primeiro Pontal & $\begin{array}{c}-------- \\
\end{array}$ & $\begin{array}{l}\text { S. densiflora, J. kraussii, Paspalum vaginatum, } \\
\text { Fimbristylis spadiceae, Salicornia gaudichaudiana, } \\
\text { Juncus acutus, Limonium brasiliensis, Sesuvium } \\
\text { portulacastrum, Phyla canescens }\end{array}$ \\
\hline & 02 & Pontal Deprec & S. alterniflora, Scirpus olneyi & $\begin{array}{l}\text { J. kraussi, J. acutus, S. olneyi, F. spadiceae, } P . \\
\text { vaginatum, Bacopa monnieri, S. gaudichaudiana, } \\
\text { Imperata brasiliensis, Axonopus sp. }\end{array}$ \\
\hline & 03 & Ponta dos Pescadores & S. alterniflora & $\begin{array}{l}\text { J. kraussi, S. densiflora, S. gaudichaudiana, L. } \\
\text { brasiliensis, P. vaginatum, Acrostichum danaefolium }\end{array}$ \\
\hline & 04 & Farol & S. alterniflora, S. densiflora & J. kraussii, S. densiflora, J. acutus \\
\hline & 05 & SJN Sul & S. alterniflora, S. densiflora & $\begin{array}{l}\text { J. kraussii, S. densiflora, J. acutus, S. maritimus, S. } \\
\text { gaudichaudiana, S. olneyi }\end{array}$ \\
\hline & 06 & Mendanha & S. olneyi, P. vaginatum & $\begin{array}{l}\text { J. kraussi, S. densiflora, S. olneyi, S. maritimus, } P . \\
\text { vaginatum, B. monnieri, Apium sp., A. danaefolium, S. } \\
\text { californicus }\end{array}$ \\
\hline & 07 & Ilha dos Ovos & S. olneyi & S. maritimus, S. olneyi, Senecio tweedii \\
\hline & 08 & Ponta Rasa & S. olneyi & $\begin{array}{l}\text { S. densiflora, S. maritimus, S. olneyi, Axonopus sp., } P \text {. } \\
\text { vaginatum }\end{array}$ \\
\hline \multirow{15}{*}{$R G$} & 09 & Molhe Oeste & S. alterniflora, S. densiflora & $\begin{array}{l}\text { S. densiflora, F. spadiceae, J. acutus, J. kraussii, S. } \\
\text { maritimus, S. gaudichaudiana }\end{array}$ \\
\hline & 11 & Mangueira Norte & S. alterniflora, Typha domingensis & J. kraussii, S. maritimus, S. densiflora, T. domingensis \\
\hline & 12 & Lagoa Verde & S. alterniflora, S. olneyi & $\begin{array}{l}\text { J. kraussii, S. maritimus, J. acutus, S. olneyi, Scirpus } \\
\text { californicus }\end{array}$ \\
\hline & 13 & Mangueira Sul & S. alterniflora, S. olneyi & J. kraussii, S. olneyi, T. domingensis \\
\hline & 14 & Rio Grande & S. alterniflora & S. maritimus, T. domingensis \\
\hline & 15 & Ilha das Pombas/Pólvora/Cavalos & S. alterniflora, S. maritimus & $\begin{array}{l}\text { S. densiflora, S. maritimus, J. kraussii, S. olneyi, S. } \\
\text { alterniflora, Myrsine parvifolia, S. tweedii, Rumex sp. }\end{array}$ \\
\hline & 16 & Justino & S. alterniflora, S. olneyi & J. kraussii, S. maritimus, S. olneyi \\
\hline & 17 & Arraial & S. alterniflora, S. olneyi & $\begin{array}{l}\text { J. kraussii, S. olneyi, S. densiflora, S. maritimus, } P . \\
\text { vaginatum, S. gaudichaudiana, A. danaefolium, Apium } \\
\text { sp. }\end{array}$ \\
\hline & 18 & Quitéria & S. alterniflora, S. olneyi & $\begin{array}{l}\text { J. kraussii, S. densiflora, S. maritimus, S. olneyi, A. } \\
\text { danaefolium }\end{array}$ \\
\hline & 19 & Arraial & --------- & J. kraussii, S. densiflora, S. olneyi, S. maritimus \\
\hline & 20 & Ilha dos Marinheiros Norte & $\begin{array}{l}\text { S. alterniflora, S. olneyi, S. } \\
\text { maritimus, S. californicus }\end{array}$ & $\begin{array}{l}\text { S. densiflora, S. maritimus, J. kraussii, Myrsine } \\
\text { parvifolia, J. acutus, A. danaefolium, S. californicus, S. } \\
\text { olneyi }\end{array}$ \\
\hline & 21 & Ilha dos Marinheiros Sul & S. alterniflora, S. olneyi & $\begin{array}{l}\text { S. densiflora, S. maritimus, J. kraussii, T. domingensis, } \\
\text { S. alterniflora }\end{array}$ \\
\hline & 22 & $\begin{array}{lr}\text { Ilha } & \text { dos } \\
\text { Carneiros/Cabras/Baicurus/Zumbi }\end{array}$ & 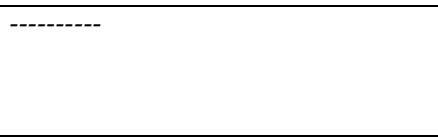 & $\begin{array}{l}\text { S. maritimus, S. densiflora, S. olneyi, J. kraussii, S. } \\
\text { gaudichaudiana, A. danaefolium, L. brasiliensis, } T \text {. } \\
\text { domingensis, Rumex sp., Cladium jamaicense,, } M . \\
\text { parvifolia }\end{array}$ \\
\hline & 23 & Ilha da Torotama & S. olneyi & S. densiflora, J. kraussii, S. olneyi \\
\hline & 24 & Pesqueiro & S. alterniflora, S. olneyi & $\begin{array}{l}\text { S. densiflora, J. kraussii, S. maritimus, J. acutus, } P \text {. } \\
\text { vaginatum, Axonopus sp., Eryngium sp., B. monnieri, } \\
\text { S. alterniflora }\end{array}$ \\
\hline
\end{tabular}




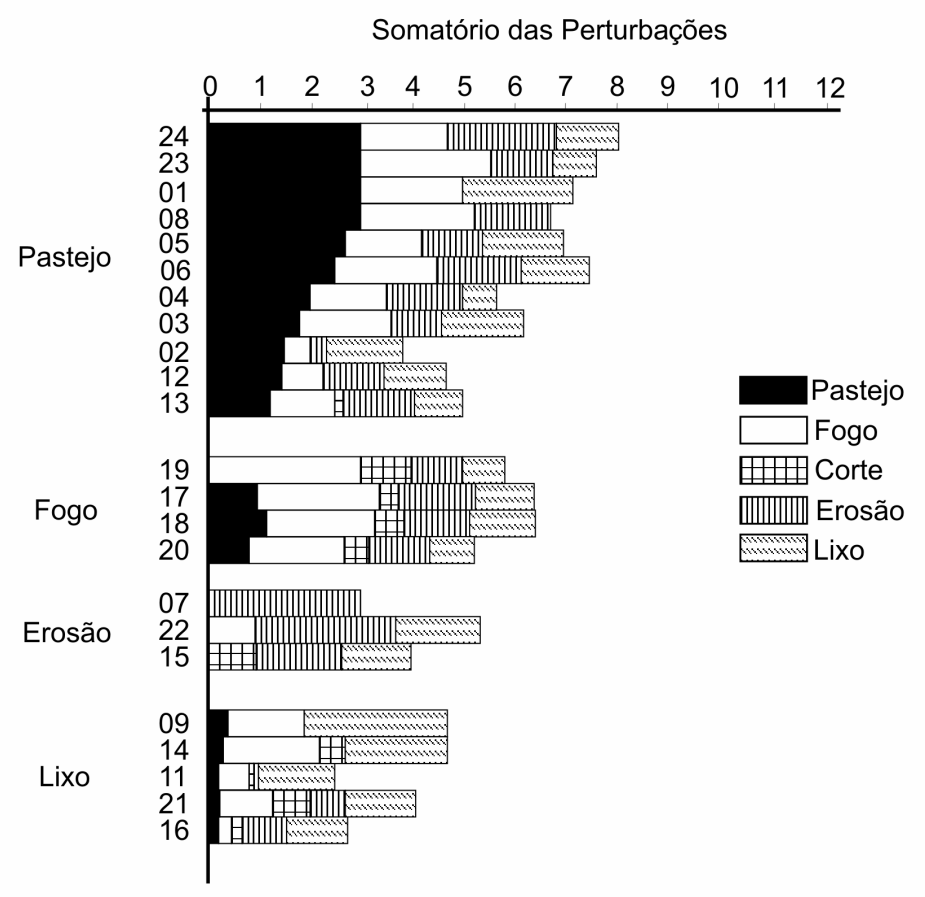

Figura 4. Somatório dos valores médios das perturbações antrópicas e natural em cada uma das 23 unidades de marisma no estuário da Lagoa dos Patos. A contribuição de cada perturbação é discriminada através das diferentes padrões de cores. A organização em grupos de unidades segue a distribuição da análise de agrupamento. A ordenação dentro dos grupos de unidades está em ordem decrescente do nível de perturbação.

A incidência de fogo atingiu a maioria das unidades de marisma (91\%), atuando com nível severo nas unidades 17, 18 e 19 em RG. Os incêndios são mais freqüentes nas marismas médias/superiores densamente vegetadas por J. kraussii e $S$. densiflora (Tabelas 3 e 4) (Figuras 2, 3 e 4).

$\mathrm{O}$ acúmulo de lixo foi observado na maioria das unidades $(91 \%)$, contudo afeta em nível severo as marismas no entorno da área urbana de RG (unidades 09 e 14). O lixo ocorre com alta freqüência tanto nas marismas inferiores (80\%) quanto nas médias/superiores (90\%) (Tabela 3) (Figuras 2, 3 e 4). Foi possível distinguir duas origens do lixo. Uma origem proveniente principalmente da atividade urbana, sendo depositada diretamente por moradores na unidade 09, composta por restos de construção, ferros, madeira, móveis, latas, papel, vidros, plásticos e material orgânico. A outra origem do lixo é da atividade urbana, portuária e pesqueira, lançando material deriva na coluna d'água, sendo posteriormente depositado na maioria das marismas, principalmente nas unidades 11, 16 e 21, com maior severidade na unidade 14 .

A erosão das margens foi constatada em 20 unidades $(87 \%)$, sendo que as pequenas ithas estuarinas ao norte de SJN (Ilha dos Ovos e Arvoredo; unidade 07) e ao norte da llha dos Marinheiros (unidade 22) demonstraram um nível severo desta perturbação (Tabela 3) (Figuras 2, 3 e 4).

O corte da vegetação foi constatado em $43 \%$ das unidades, sendo um impacto de nível baixo, exclusivo sobre marismas médias/superiores, se concentrando na região central do estuário, sobre as unidades $15,20 \mathrm{e}$ 21 vegetadas por $S$. densiflora e 18 e 19 ocupadas por J. kraussii (Tabelas 3 e 4) (Figuras 2, 3 e 4).

Foi constatada a presença de aterros/canais de drenagem em $52 \%$ das unidades e a descontinuidade da cobertura vegetal halófita das marismas em 6 unidades (26\%). Os canais de drenagem e aterros se caracterizam por possuírem pequena largura ( $>1$ até $3 \mathrm{~m}$ ) (Tabela 4$)$.

\section{DISCUSSÃO}

A distribuição setorizada das atividades antrópicas no estuário da Lagoa dos Patos resulta em uma variação entre as unidades de marisma quanto ao tipo e severidade das perturbações a que estão sujeitas. 


\section{Pastejo}

O pastejo por gado bovino (corte e leite) e equino atua mais intensamente sobre as marismas em São José do Norte e na llha da Torotama e Pesqueiro, em Rio Grande. As plantas mais freqüentemente pastadas são S. olneyi e S. densiflora. Marangoni (2008) constatou que o consumo de $S$. olneyi por bovinos pode ocorrer $\mathrm{o}$ ano todo, sendo intensificado durante a primavera e o verão, enquanto o pastejo sobre $S$. densiflora se dá principalmente durante o inverno.

O pastejo pode estar afetando alguns processos biológicos e ecológicos nas marismas locais, alterando a composição vegetal, a exportação de matéria orgânica (detrito) e a reciclagem de nutrientes. A ocorrência de gramas de pequeno porte como Axonopus sp (espécie comum em campos; Kissman 1997) e P. vaginatum (unidades 02, 04, 06, 08 e 24) indicam um pastejo freqüente sobre as plantas dominantes de maior porte $(S$. densiflora, $S$. alterniflora e $S$. olneyi). Algumas espécies do gênero Axonopus toleram o pisoteio, sendo resistentes a cortes e queimadas, bem como a períodos de estiagem e inundação (Kissman 1997). P. vaginatum é uma planta com grande amplitude ecológica (Costa \& Marangoni 2000), anteriormente já utilizada como indicadora de áreas de marismas sujeitas a pastejo (Costa et al. 1997). Conseqüentemente, a ausência de competidores de maior porte favorece a expansão de ambas espécies vegetais sobre algumas marismas. Similarmente Miller et al. (1997) constataram a invasão de $P$. vaginatum em áreas de Scirpus americanus pastadas por ganso nos EUA.

Quanto à exportação de matéria orgânica, o pastejo pode estar atuando de forma diferenciada sobre as marismas vegetadas por $S$. olneyi e $S$. densiflora. As marismas inferiores (alagada 40-60\% do tempo) é um importante contribuinte de detrito para o estuário da Lagoa dos Patos (Peixoto 1997; Cunha et al. 2005; Coimbra 2008), assim o consumo de $S$. olneyi poderia estar reduzindo a quantidade de matéria orgânica exportada para a região estuarina e costeira. Nas marismas médias de $S$. densiflora o consumo pelo gado pode ter pouca interferência, pois apenas uma pequena parcela da biomassa produzida é exportada, sendo a maior parte decomposta in situ ou consumida por macroconsumidores (e.g. isópodes terrestres, caranguejo Neohelixchasmagnathus granulata) (D'Incao 1990, Gaona et al. 1996, Costa et al. 1997, Costa 1998, Seeliger 2001, Costa et al. 2003, Peixoto \& Costa 2004).

A presença de gado pode não estar prejudicando o ciclo de nutrientes nas marismas, pois apesar da remoção de biomassa vegetal, os animais produzem dejetos (fezes e urina) com uma composição química de até $90 \%$ do $N$ oriundo da vegetação pastada, participando desta maneira na reciclagem de nutrientes (Moot \& Poeponoe 1977, Cantarutti et al. 2001). A magnitude da interferência dos animais na reciclagem dos nutrientes dependerá da distribuição das excreções animais na pastagem e da área afetada pelas excreções e pelos seus teores de nutrientes, este sendo dependente da dieta alimentar e/ou forma bacteriana no trato digestivo (Lysyk et al. 1985, Cantarutti et al. 2001). A interação positiva entre herbívoros e plantas das marismas vem sendo estudada na costa subártica canadense, onde o consumo por gansos selvagens migratórios tem demonstrado um aumento na produção de biomassa vegetal. Este efeito positivo é devido ao enriquecimento de nitrogênio pelas excreções e à contínua atividade de crescimento das monocotiledôneas de crescimento basal pastadas (constante renovação) (Cargill \& Jefferies 1984, Bazely \& Jefferies 1985).

Tanto $S$. olneyi quanto $S$. densiflora possuem boa capacidade de recuperação após pastejo, ocorrendo principalmente através da produção vegetativa via rebrote dos rizomas. Scirpus americanus ( $=S$. olneyi) consegue recuperar a biomassa em até 2 anos após o pastejo por gansos na América do Norte e Canadá (Giroux \& Bédard 1987, Miller et al. 1997). Entretanto gansos pastam preferencialmente os rizomas de $S$. americanus prejudicando a produção de biomassa, diferentemente da perturbação pelo gado nas marismas locais, a qual atua exclusivamente sobre a parte área, sendo provável a recuperação em um período menor do que 2 anos. Silva et al. (1993) verificou a rápida recuperação de biomassa aérea de $S$. densiflora após 6 meses de remoção mecânica (corte).

\section{Fogo}

O fogo demonstrou ser um impacto amplamente difundido nas marismas, sendo mais freqüente sobre as marismas superiores de $J$. kraussii e médias de $S$. densiflora, nas proximidades de estradas ou de 
propriedades agropecuárias. Os incêndios são mais comuns durante o período do verão e início do outono, ocorrendo próximo às estradas (descarte de cigarros ou fósforos acesos; Costa \& Marangoni 2000), em acampamentos de verão dos pescadores artesanais e em propriedades rurais com o propósito de renovação dos campos ou eliminação de plantas indesejáveis (Marangoni 2008). A disponibilidade de grandes quantidades de biomassa vegetal sobre a superfície em marismas dominadas por J. kraussii (até 4.500 $\mathrm{gPS} / \mathrm{m}^{2}$; Gaona et al. 1996) e S. densiflora (até 2.700 $\mathrm{gPS} / \mathrm{m}^{2}$; Silva et al. 1996, Peixoto \& Costa 2004), associada à baixa freqüência de alagamento (3 - 20\% do tempo; Costa 1998), aumentam consideravelmente a vulnerabilidade destas áreas a incêndios durante os meses menos chuvosos (verão e outono) no estuário da Lagoa dos Patos.

Os incêndios nas marismas geralmente atingem a cobertura vegetal, sem danos maiores sobre rizomas e raízes das plantas, possibilitando uma rápida recuperação da cobertura vegetal. Figueroa et al. (1988) e Nieva \& Figueroa (1997) verificaram uma rápida produção de biomassa de $S$. densiflora após fogo, na Espanha, alcançando uma taxa média de recuperação de $64 \%$ ao ano, fazendo com que uma área queimada seja restabelecida em menos de 2 anos. Isacch et al. (2004) verificaram a recuperação na estrutura da comunidade vegetal de $J$. acutus após 1 ano de um incêndio, similar ao relatado em marismas vegetadas por Juncus roemerianus nos EUA (Schmalzer et al. 1991). Schmalzer et al. (1991) e Hackney \& De La Cruz (1981) estudando a ação do fogo sobre J. roemerianus nos EUA, verificaram que a recuperação da biomassa aérea ocorre em 3 anos após o incêndio.

A remoção da densa cobertura das plantas dominantes de marisma ( 1,0 a $1,8 \mathrm{~m}$ de altura) pelo fogo pode permitir a colonização de outras espécies, tais como Vigna luteola, Senecio tweedii e Aster squamatus (Costa \& Marangoni 2000). Áreas de S. densiflora queimadas na Argentina aumentaram o número de espécies, favorecido pela diminuição da cobertura-abundância das plantas perenes dominantes (Madanes et al. 2007). Isacch et al. (2004) e Madanes et al. (2007) constataram que a configuração original da comunidade de $S$. densiflora não retornou ao estado de pré-perturbação mesmo um ano após o incêndio.

\section{Lixo}

O acúmulo de lixo sobre as marismas é determinado por processos ativos e passivos do material descartado ou carreado pelo vento $e$ escoamento pluvial no estuário da Lagoa dos Patos.

Um grande depósito de lixo foi constatado nas áreas mais elevadas da marisma do Molhe Oeste (processo ativo) (unidade 09), apesar de ser uma Área de Preservação Ambiental do Porto do Rio Grande (SUPRG 2008). Nesta marisma são previstas atividades de turismo e lazer, orientadas sob um plano de manejo ambiental, contudo, na prática é uma área de livre acesso ao público (e.g. pesca esportiva, acampamento, lazer), servindo principalmente como área de despejo.

Diferentemente, a deposição do lixo em deriva (processo passivo), que chega ao estuário pelo escoamento pluvial ou perdas nas operações portuárias, afeta as marismas inferiores e médias, sendo sua distribuição dependente da variação no nível de água da Lagoa. Azevedo (2000) constatou que nas marismas locais, o acúmulo do material em deriva é mais freqüente nas marismas com alturas próximas ao nível médio da água da Lagoa $(+15 \mathrm{~cm}$; $\sim 40 \%$ do tempo alagado), podendo ocorrer em áreas mais elevadas inundadas em períodos de águas excepcionalmente altas na Lagoa $(+27 \mathrm{~cm}$; zonas alagadas menos que $12 \%$ do tempo).

A presença de plantas oportunistas (e.g. Senecio tweedii, A. danaefolium, Apium sp. e Rumex sp.) em áreas de deposição de material de deriva foi constatada em várias marismas, com acentuada ocorrência nas margens das unidades 15 (llha da Pólvora) e 17 (Leonídeo). Conforme Costa et al. (1997) e Azevedo (2000) estas espécies vegetais são indicadoras de brechas na cobertura vegetal dominante e deposição de lixo orgânico. Azevedo (2000) identificou 55 espécies de plantas nas marismas locais, sendo 17 (31\%), com ciclo de vida anual ou bianual encontradas exclusivamente nas áreas perturbadas pela deposição de lixo.

A deposição do material sobre a vegetação dominante das marismas resulta, na maioria das vezes, na morte das plantas dominantes e conseqüente abertura de brechas (Reidenbaugh \& Banta 1980, Adam 1990), sendo geralmente colonizadas por plantas oportunistas, incomuns nas 
áreas naturais não perturbadas (Bertness \& Ellison 1987, Bertness 1999). O processo de recuperação da cobertura vegetal pelas espécies dominantes ocorre entre 1 a 3 anos após a perturbação, através dos rizomas vindos das margens da brecha ou pela germinação de sementes (Bertness \& Ellison 1987, Azevedo 2000).

\section{Erosão}

A avaliação visual da perturbação das marismas por erosão assinala o processo erosivo como mais severo quando ocorrendo sobre as marismas médias/superiores, gerando na maioria das vezes um escarpamento das margens. As unidades assinaladas como sofrendo erosão foram às mesmas verificadas através do mapeamento das marismas nas últimas 5 décadas por Marangoni (2008), o qual quantificou uma taxa média de erosão sobre as ilhas estuarinas de 1,3\% por ano (unidades 07 e 22). É importante comentar que a avaliação visual de impacto é um método eficiente para a detecção, mas não para o dimensionamento dos efeitos erosivos sobre as marismas, assim a utilização de sensoriamento remoto é indispensável para a correta quantificação desta perturbação natural.

\section{Corte}

O corte da vegetação para a atividade agrícola demonstrou ser uma perturbação restrita a llha dos Marinheiros e ilhas próximas, Quitéria e Arraial. Este impacto é ocasionado exclusivamente por pequenos agricultores que cortam com maior freqüência $S$. densiflora e J. kraussii. Seeliger \& Costa (1998) descrevem esta perturbação como de pequena escala $\left(2-20 \mathrm{~m}^{2}\right)$, entretanto, no presente estudo foram encontradas áreas cortadas com 800 e até $1.900 \mathrm{~m}^{2}$.

Azevedo (2000) observou que as brechas abertas pelo corte, em uma área de $S$. densiflora, são recompostas em 2 a 3 anos. Silva et al. (1993) constataram a rápida produção de biomassa de $S$. densiflora, após 6 meses da remoção da biomassa aérea, encontrando valores similares de biomassa das áreas sem corte (Peixoto \& Costa 2004). Marangoni (2008) relata que o corte de $S$. densiflora é uma atividade que vem ocorrendo nos últimos 270 anos, entretanto tem sido verificada uma substancial diminuição desta atividade pelos agricultores.

\section{Canais de Drenagem, Aterros e Descontinuidade Cobertura Halófitas}

Das perturbações antrópicas estimadas apenas qualitativamente, os canais de drenagem e aterros são perturbações responsáveis pela supressão total da cobertura vegetal. Os canais de drenagem na zona rural do estuário da Lagoa dos Patos são construídos com o intuito de drenar as áreas cultivadas e para facilitar o acesso das pequenas embarcações dos agricultores e pescadores ao estuário (Costa et al. 1997). Na área urbana os pequenos canais servem para escoamento de água pluvial e esgoto doméstico. Nas áreas rurais a maior parte dos aterros são construídos contíguos aos canais de drenagem, já na zona urbana os aterros são comuns em margens erosivas, sendo utilizados entulho de obras, pneus e madeira. Neste estudo foi constatada a ocorrência de canais de drenagem e aterros em 12 unidades de marisma, configurando 3 unidades a mais (Illha da Torotama e Pesqueiro em RG e Mendanha em SJN) do que o relatado por Costa (1997), Costa et al. (1997) e Costa \& Marangoni (2000). Nestas unidades, os canais foram construídos essencialmente para impedir o avanço do gado ao estuário ou a propriedade vizinha (RG) e para captação de água e emissão de efluentes para aqüicultura (SJN).

A descontinuidade da cobertura vegetal halófita dentro das marismas com a dominância de Typha domingensis e Scirpus californicus ocorrem em locais de baixa salinidade, como em desembocadura de brejos/banhados, canais de drenagem, escoamento pluvial e esgoto. Zedler et al. (1990) constatou a exclusão competitiva de J. kraussii por Typha orientalis, na Austrália, sob baixas salinidades (5-10), sendo esta interação revertida com o aumento da salinidade (20-40). Conseqüentemente, um aumento no número de canais de drenagem, devido a maior demanda urbana (escoamento pluvial) e rural (agricultura, aqüicultura), bem como, possíveis mudanças hidrológicas no estuário (e.g. diminuição da salinidade) podem ser responsáveis pelas modificações observadas na comunidade vegetal das marismas.

\section{CONCLUSÕES}

As marismas do estuário da Lagoa dos Patos apresentaram perturbações antrópicas e/ou de origem natural, que de forma geral afetam a cobertura vegetal com baixa intensidade e tem moderada extensão (10-50 m). 
O pastejo, fogo e corte da vegetação foram as principais perturbações oriundas da atividade agropecuária e acúmulo de lixo da atividade urbana.

As perturbações antrópicas vêm causando modificações temporárias (pastejo, fogo, lixo, corte) e permanentes (canais de drenagem e aterros) principalmente na estrutura da comunidade vegetal das marismas, ao favorecerem a ocupação e crescimento de outras plantas (oportunistas e glicófitas como $T$. domingensis e $S$. californicus), todavia este ecossistema se caracteriza por uma excelente capacidade de resiliência.

\section{LITERATURA CITADA}

ABREU, PC, CSB COSTA, C BEMVENUTI, C ODEBRECHT, W GRANÉLI \& AM ANÉSIO. 2006. Eutrophication Processes and Trophic Interactions in a Shallow Estuary: Preliminary Results Based on Stable Isotope Analysis ( $\delta 13 \mathrm{C}$ and $\delta 15 \mathrm{~N})$. Estuaries and Coasts., 29(2): 277-285.

ADAM, P. 1990. Saltmarsh Ecology. New York, Cambridge University Press. 461p.

AZEVEDO, AMG. 2000. Hábitats, associações vegetais e fenologia das plantas das marismas da llha da Pólvora, Estuário da Lagoa dos Patos (RS, Brasil). Dissertação de Mestrado. Programa de PósGraduação em Oceanografia Biológica - FURG, Rio Grande. 102 p.

BAKKER, JP, D BOS \& Y DE VRIES. 2003. To graze or not to graze: that is the question. In: Wolff, WJ (ed.). Challenges to the Wadden Sea area. Proceedings of the 10th international scientific Wadden Sea symposium, Groningen 2000. LNV Noord, Groningen, 67-87. Disponível em: http://home.planet.nl/ daan.bos/papers/bakker_etal_2003_iwss .pdf. Acesso em: 19/03/2008.

BAZELY, DR \& RL JEFFERIES. 1985. Goose faeces: a source of nitrogen for plant growth in a grazed salt marsh. Journal of Applied Ecology., 22: 693-703.

BEMVENUTI, CE. 1998. Estrutura Trófica. In: SEELIGER, U, C ODEBRECHT \& JP CASTELLO (ed.). Os Ecossistemas Costeiro e Marinho do Extremo Sul do Brasil. Editora Ecoscientia, Rio Grande, Cap. 5.2: 79-82.

BERTNESS, MD. 1999. The ecology of Atlantic shorelines. Massachusetts. Sinauer Associates, Inc. 417 p.

BERTNESS, MD \& AM ELLISON. 1987. Determinants of Pattern in a New England Salt Marsh Plant Community. Ecological Monographs., 57(2): 129-147.

BOS, D, JP BAKKER, Y DE VRIES \& S VAN LIESHOUT. 2002. Long-term vegetation changes in experimentally grazed and ungrazed back-barrier marshes in the Wadden Sea. Applied Vegetation Science., 5: 45-54.

BREWER, JS, JM LEVINE \& MD BERTNESS. 1998. Interactive effects of elevation and burial with wrack on plant community structure in some Rhode Island salt marshes. Journal of Ecology., 86(1): 125-136.

CANTARUTTI, RB, D NASCIMENTO, OV COSTA. 2001. Impacto do animal sobre o solo: Compactação e reciclagem de
A ordenação das unidades de marisma quanto ao tipo e grau das perturbações antrópicas e erosão, causadores de modificações na cobertura vegetal, pode ser um importante instrumento para o desenvolvimento de propostas/projetos de gerenciamento ambiental mais adequado à realidade de cada unidade.

\section{AGRADECIMENTOS}

Este estudo foi financiado através de Bolsa de PósGraduação Nível Doutorado fornecida pelo CNPq (Ministério da Ciência e Tecnologia) (Processo no 141797/2004-5 (JCM) e 301763/2006-2 (CSBC).

nutrientes. In: MATTOS, WRS, Produção animal na visão dos brasileiros. Fundação de Estudos Agrários "Luiz de Queiroz", Piracicaba, 826-837.

CARGILL, SM \& RL JEFFERIES. 1984. The effects of grazing by lesser snow geese on the vegetation of a sub-arctic salt marsh. Journal of Applied Ecology., 21: 669-686.

COIMBRA, FL. 2008. Avaliação da dinâmica espectral das marismas da Lagoa dos Patos (RS) mediante utilização de imagens CCD/CBERS-2. Dissertação de Mestrado, Fundação Universidade Federal do Rio Grande, Brasil, $128 \mathrm{p}$.

COSTA, CSB. 1997. Vegetação. In: TAGLIANI, PRA \& ML ASMUS (coord.). Estudo de Impacto Ambiental do Porto de Rio GrandeRS. Relatório Final. Cap. 4.2.2.4: 350-403. Disponível em: http://www.labgerco.furg.br/file/pdf/Impacto_Ambiental_Porto_R io_Grande.pdf. Acesso em: 11/11/2007.

COSTA, CSB. 1998. Marismas Irregularmente Alagadas. In: SEELIGER, U, C ODEBRECHT \& JP CASTELLO (ed.). Os Ecossistemas Costeiro e Marinho do Extremo Sul do Brasil. Editora Ecoscientia, Rio Grande, Cap. 5.3: 82-87.

COSTA, CSB \& AJ DAVY. 1992. Coastal saltmarsh communities of Latin America. In: SEELIGER, U (ed.). Coastal plant communities of Latin America. Academic Press, Inc., California, Cap. 12: 179-198.

COSTA, CSB \& JC MARANGONI. 2000. Impacto Ambiental do asfaltamento da BR101 sobre as marismas de São José do Norte (RS, Brasil): Estado atual e efeitos potenciais. In: Anais do V Simpósio de Ecossistemas Brasileiros. 10-15 de outubro de 2000. ACIESP. São Paulo, Vol. 1: 268-291. Disponível em:

http://www.peld.furg.br/grp/ccosta/Anais/Costa\&Marangoni2000.pdf . Acesso em: 14/03/2007.

COSTA, CSB, JC MARANGONI \& AMG AZEVEDO. 2003. Plant zonation in irregularly flooded salt marshes: relative importance of stress tolerance and biological interactions. Journal of Ecology., 91: 951-965.

COSTA, CSB, U SEELIGER, CPL OLIVEIRA \& AMM MAZO. 1997. Distribuição, funções e valores das marismas e pradarias submersas no Estuário da Lagoa dos Patos (RS, Brasil). Atlântica., 19: 67-85.

CUNHA, SR, M ASMUS \& CSB COSTA. 2005. Production dynamics of Spartina alterniflora salt marshes in the estuary of Patos Lagoon (RS, Brazil): A simulation model approach. Braz. J. Aquat. Sci. Technol., 9(2):75-85. 
D'INCAO, F, KG SILVA, ML RUFFINO \& AC BRAGA. 1990. Hábito alimentar do caranguejo Chasmagnathus granulata Dana, 1851 na Barra do Rio Grande, RS (Decapoda, Grapsidae). Atlântica., 12(2): 85-93.

DORMANN, CF \& JP BAKKER. 2000. The Impact of Herbivory and Competition on Flowering and Survival during Saltmarsh Succession. Plant Biol., 2: 68-76.

FELDMAN, SR \& JP LEWIS. 2005. Effects of fire on the structure and diversity of a Spartina argentinensis tall grassland. Applied Vegetation Science., 8: 77-84.

FIGUEROA, ME, MJ MARTOS, MR LIÑAN \& JMF PALACIOS. 1988. Aspectos bioclimáticos de la ecologia de Spartina marítima (Curt.) Fern. y Spartina densiflora Brong. en las marismas de Huelva (SO, Espana). Avances en Investigación Bioclimatologia - CSIC. Madrid. 243-250.

GAONA, CAP, AR PEIXOTO \& CSB COSTA. 1996. Produção Primária de uma marisma raramente alagada dominada por Juncus effusus L., no extremo sul do Brasil. Atlântica., 18: 43-54.

GIROUX, JF \& J BÉDARD. 1987. The effects of grazing by greater snow geese on the vegetation of tidal marshes in the St. Lawrence Estuary. Journal of Applied Ecology., 24: 773-788.

HACKNEY, CT \& AA DE LA CRUZ. 1981. Effects of fire on brackish marsh communities: management implications. Wetland Management., 1(1): 75-86.

HAIR, JF, RE ANDERSON, RL TATHAM \& WC BLACK. 2005. Análise Multivariada de Dados. 5 Ed. Porto Alegre. Bookman. 593p.

HAMMER, O, DAT HARPER \& PD RYAN. 2001. Past: Paleontological Statistics Software Package for Education and Data Analysis. Palaeontologia Electronica., 4(1): 1-9. Disponível em: http://palaeoelectronica.org/2001_1/pastissue1_01.htm. Acesso em: 14/07/2008.

HOWELL, DC. 2001. Resampling Procedures Version 1.3. Disponível em:http://www.uvm.edu/ dhowell/StatPages/Resampling/Resamplin gPackage.zip. Acesso em: 29/07/2008.

ISACCH, JP, S HOLZ, L RICCI \& MM MARTINEZ. 2004. Post-fire vegetation change and bird use of a salt marsh in coastal Argentina. Wetlands., 24(2): 235-243.

KISSMANN, KG. 1997. Plantas Infestantes e Nocivas. TOMO I. 늘. São Paulo, BASF Brasileira S.A. 606p.

LYSYK, TJ, ER EASTON \& PD EVENSON. 1985. Seasonal changes in nitrogen and moisture content of cattle manure in cool-season pastures. Journal of Range Management., 38(3): 251-254.

MADANES, N, S FISCHER \& R VICARI. 2007. Efectos del fuego en una marisma de Spartina densiflora en la planicie de inundación del río Paraná, Argentina. Rev. Chil. Hist. Nat. [online]., 80(2): 187-199. Disponível

em: http://www.scielo.cl/scielo.php?script=sci_arttext\&pid=S0716078X2007000200005\&lng=es\&nrm=iso. Acesso em: 09/02/2008.

MARANGONI, JC. 2003. Caracterização da paisagem de uma área de preservação: Estudo de caso da lagoinha (Rio Grande, RS). Atlântica., 25(2): 163-169.

MARANGONI, JC. 2008. Subsídios para o gerenciamento das marismas no estuário da Lagoa dos Patos (RS). Tese de Doutorado. Programa de Pós-Graduação em Oceanografia Biológica - FURG, Rio Grande. 141 p.

MOOT, GO, HL POPENOE. 1977. Grasslands. In: ALVIM, PT \& TT. KOZLOWSKI (ed). Ecophysiology of tropical crops. Academic Press, New York, Chap. 6: 157-186.
MILLER, DL, FE SMEINS, JW WEBB \& MT LONGNECKER. 1997. Regeneration of Scirpus americanus in a Texas coastal marsh following lesser snow goose herbivory. Wetlands., 17(1): 31-42.

NIEVA, FJJ \& ME FIGUEROA. 1997. Patron de recuperacion trás fuego en uma marisma de Spartina densiflora Brong. In: VII COLACMARCongresso Latino-americano sobre Ciências do Mar. Santos. 2226/09/1997. Resumos Expandidos 2: 213-214.

NOGUEIRA, RXS \& CSB COSTA. 2003. Mapeamento das marismas do estuário da Lagoa dos Patos (RS) utilizando fotografias aéreas digitais $35 \mathrm{~mm}$ no modo infravermelho. In: Congresso da Associação Brasileira de Estudos do Quartenário-ABEQUA. Recife. Resumos Expandidos 9. Disponível em: <http://www.peld.furg.br/grp/ccosta/Anais/Nogueira\&Costa2003b.PD F>. Acesso em: 20/01/2007.

PEIXOTO, AR. 1997. Análise Simultânea da Produção Primária das Comunidades de Macrófitas Emergentes Dominantes das Marismas do Estuário da Lagoa dos Patos (RS) - Brasil. Tese de mestrado. UFRGS, Porto Alegre (RS). $112 \mathrm{p}$.

PEIXOTO, AR \& CSB COSTA. 2004. Produção primária líquida aérea de Spartina densiflora Brong. (Poacea) no estuário da Lagoa dos Patos, Rio Grande do Sul, Brasil. Iheríngia, Ser. Bot., 59(1): 27-34.

REIDENBAUGH, TG \& WC BANTA. 1980. Origin and effects of Spartina wrack in a Virginia salt marsh. Gulf Research Reports., 6(4): 393-401.

ROMESBURG, HC. 1984. Cluster Analysis for Researchers. USA, Lifetime Learning Publications. 334p.

SCHMALZER, PA, CR HINKLE \& JL MAILANDER. 1991. Changes in community composition and biomass in Juncus roemerianus Scheele and Spartina bakeri Merr. Marshes one year after a fire. Wetlands., 11(1): 67-86.

SEELIGER, U. 2001. The Patos Lagoon Estuary, Brazil. In: SEELIGER, U \& B KJERVE (ed.). Coastal Marine Ecosystems of Latin America. Springer-Verlag, Berlin, Cap. 12: 167-183.

SEELIGER, U, CSB COSTA \& PC ABREU. 1998. Fluxo de Energia e Hábitats no Estuário da Lagoa dos Patos. In: SEELIGER, U, C ODEBRECHT \& JP CASTELLO (ed.). Os Ecossistemas Costeiro e Marinho do Extremo Sul do Brasil. Editora Ecoscientia, Rio Grande, Cap. 5.1: 73-78.

SEELIGER, U \& CSB COSTA. 1998. Impactos Naturais e Humanos. In: SEELIGER, U, C ODEBRECHT \& JP CASTELLO (ed.). Os Ecossistemas Costeiro e Marinho do Extremo Sul do Brasil. Editora Ecoscientia, Rio Grande, Cap. 10: 217-226.

SILVA, CP, CMP PEREIRA \& LPP DORNELES. 1993. Espécies de gramíneas e crescimento de Spartina densiflora Brong. em uma marisma da Laguna dos Patos, RS, Brasil. Cad. Pesq. Ser. Bot. (Santa Cruz do Sul)., 5(1): 95-108.

SUPRG. 2008. Plano de zoneamento das áreas do porto organizado de Rio Grande. Disponível em: http://www.portoriogrande.com.br/pt/instalacoes/zoneamento_p orto.pdf. Acesso em: 01/09/2008)

TURNER, MG. 1987. Effects of grazing feral horses, clipping, trampling and burning on a Georgia marsh. Estuaries., I: 54-60.

ZEDLER, JB, E PALING \& A MCCOMB. 1990. Differential responses to salinity help explain the replacement of native Juncus kraussii by Typha orientalis in Western Australian salt marshes. Australian Journal of Ecology., 15: 57-72.

Recebido: 10/10/2008

Aceito: 16/03/2009 